\title{
Direct-To-Satellite IoT - A Survey of the State of the Art and Future Research Perspectives
}

\section{Backhauling the IoT through LEO Satellites}

\author{
Juan A. Fraire ${ }^{1[0000-0001-9816-6989]}$, Sandra Céspedes ${ }^{2[0000-0003-1686-2644]}$, \\ and Nicola Accettura ${ }^{3[0000-0002-6334-2185]}$ \\ 1 CONICET-UNC, Córdoba, Argentina \\ Dipartimento di Elettronica, Politecnico di Torino, Torino, Italy \\ Tel.: +54-9351-2446010 \\ juanfraire@unc.edu.ar \\ 2 Department of Electrical Engineering, Universidad de Chile, Santiago, Chile, and \\ NIC Chile Research Labs, Santiago, Chile. \\ scespedes@ing.uchile.cl \\ 3 LAAS-CNRS, Université de Toulouse, CNRS, Toulouse, France \\ nicola.accettura@laas.fr
}

\begin{abstract}
The Internet of Things (IoT) has drawn an enormous attention into the scientific community thanks to unimaginable before applications newly available in everyday life. The technological landscape behind the implied surge of automated interactions among humans and machines has been shaped by plugging into the Internet very low power devices that can perform monitoring and actuation operations through very cheap circuitry. The most challenging IoT scenarios include deployments of low power devices dispersed over wide geographical areas. In such scenarios, satellites will play a key role in bridging the gap towards a pervasive IoT able to easily handle disaster recovery scenarios (earthquakes, tsunamis, and flash floods, etc.), where the presence of a resilient backhauling communications infrastructure is crucial. In these scenarios, Direct-to-Satellite IoT (DtS-IoT) connectivity is preferred as no intermediate ground gateway is required, facilitating and speeding up the deployment of wide coverage IoT infrastructure. In this work, an in-depth yet thorough survey on the state-of-the-art of DtS-IoT is presented. The available physical layer techniques specifically designed for the IoT satellite link are described, and the suitability of both the current Medium Access Control protocol and the upper layer protocols to communicate over space links will be argued. We also discuss the design of the overall satellite LEO constellation and topology to be considered in DtS-IoT networks.
\end{abstract}

Keywords: Internet of Things $\cdot$ Low-Earth Orbit Satellite $\cdot$ Low Power Wide Area Networks (LPWAN) - Satellite Networks. 


\section{Introduction}

By embedding computational capabilities into every-day objects, it is possible to easily interact with the environment and draw out complex monitoring and actuation operations in several challenging and critical application areas, such as disaster recovery, smart agriculture, and industrial processes, among others. The consequent tidal increase of communications among humans and objects has made actual the concept of pervasive computing through its natural accomplishment, the Internet of Things (IoT). Triggered by such a revolutionary networking paradigm, a huge number of concurrent technological solutions have been proposed. Some of them have been standardized [40], while others are getting momentum in recent years after some incubation time [51]. In general, each technology is tailored to provide a specific coverage, to support a target data-rate, and to transport specific message sizes.

Sensor-oriented technologies and protocols (e.g., ZigBee, 6TiSCH, and ZWave) have been designed to be used in small devices with low power consumption and with data rates in the order of hundreds of kbps. These protocols are only suitable for low traffic applications at both short range in Wireless Personal Area Networks (WPANs), and long range in multihop WPANs [40]. In contrast, Cellular Networks (e.g., UMTS and LTE) offer a rich variety of longrange, high-data rate, from tens of kbps up to several Mbps. Even though they can be used for IoT communications, traditional cellular networks were not designed for the transmission of small messages nor for low-consumption terminals. In this context, a new type of network is currently emerging: Low-Power WideArea (LPWA) Networks. Some representative LPWA technologies as LoRa [34] and SigFox [50] are specifically designed to share the properties of both WPANs and cellular networks: low-power and long-range (more than $10 \mathrm{~km}$ ). From an architectural point of view, LPWA are featured by a hierarchical network organization: a network server coordinates several gateways through a reliable backhaul; in turn, gateways are in charge of interacting through wireless links with potentially billions of low power devices. LPWA protocols are tailored for low data rate applications, from hundreds of bps up to several hundreds of kbps, with messages no longer than 100 bytes, on average [48]. As a result, LPWA transceivers can offer several years of battery autonomy and provide very cheap service on cheap terminal devices.

The long-range, low-power, and low-data properties make the LPWA technology an appealing candidate for many IoT applications. Among them, developing countries with agriculture-based economies will benefit from keeping track and monitoring crops. Based on the sensing information, smart decisions can be taken to get better productivity, with a consequent positive impact on the national economy [55]. LPWA will play a key role in the development of smart agriculture solutions [5], as well as in many other applications, including Smart Grid, Environmental Monitoring, and Emergency Management, among others [17].

Although different in many aspects, the abovementioned terrestrial technologies have a common weakness: they fail to provide global connectivity. Terres- 
trial networks are also highly vulnerable in cases of natural disasters or terrorist attacks. Instead, satellite access networks, and, in particular, Large-scale LowEarth Orbit (LEO) satellite constellations have shown their potential to extend terrestrial networks to address the above issues. In this context, satellites can be leveraged to support a world-wide expansion of the promising IoT market. Several long-range low-power satellite systems are already operational, while others are being prepared. Most of them make use of a LEO satellite constellation, such as Orbcomm, Iridium, Globalstar or Argos [46], each featured by a specific orbital formation. LEO satellites are characterized by an altitude between $160 \mathrm{~km}$ and $2000 \mathrm{~km}$ which renders a ground coverage of several hundreds of kilometers and a reasonable Round Trip Time (RTT) delay for most applications (i.e., less than $100 \mathrm{~ms}$ ) [36, 15]. Contrariwise, Geostationary Earth Orbit (GEO) satellite links induce higher RTT in the order of $600 \mathrm{~ms}$ to $700 \mathrm{~ms}[57,38]$. Recently, the combination of satellite networks and LPWA technologies has been proposed as a promising hybrid networking architecture. Indeed, backhauling satellite networks interconnecting LPWA gateways provide both reachability in remote areas and redundancy in case of service disruption of the ground network. In the case of LEO deployments, even delay sensitive applications could be served by such a hybrid architecture; instead, in GEO deployments, backhauling with LPWA gateways will target applications that do not hold critical time constraints [41]. Hence, given that we target delay-sensitive applications, including disaster recovery scenarios, the focus of the present contribution is on the interconnection of LPWA gateways through LEO satellite networks.

We first note that, depending on the network configuration, data can be directly transmitted from LEO satellites to Internet via ground stations in range, or relayed to another satellite via inter-satellite links, or stored and carried until a suitable ground station is on sight. Indeed, users from the ground see a LEO satellite crossing the sky with a very high speed (several $\mathrm{km} / \mathrm{s}$ ), and within a time interval of no more than around 10 minutes on average. Further than the implied Doppler effects on communications, such a particular configuration impacts the overall network topology dynamics, that must seamlessly permit handover from one satellite to another. Nevertheless, satellite communications provide a more cost-effective solution with respect to other terrestrial technologies [22] and have the potential to play an important role for different reasons as listed in [17]: $(i)$ smart objects are often remote or they are dispersed over a wide geographical area or they are inaccessible; $(\boldsymbol{i i})$ satellites can naturally support broadcast transmissions (i.e., towards all nodes of the whole network), multicast transmissions (i.e., towards a portion of nodes of the whole network) or geocast transmissions (i.e., towards a portion of nodes placed in a given area of the network) [25]; (iii) satellites can provide an alternative redundant path for critical applications requiring high availability at reasonable cost; and (iv) existing LPWA applications generally target low data rate transmission indicating that current low bandwidth satellite infrastructures can be effectively reused.

That being said, the most relevant scientific interest is focused on the network link between satellites and IoT devices on ground. As illustrated in Fig. 1, there 

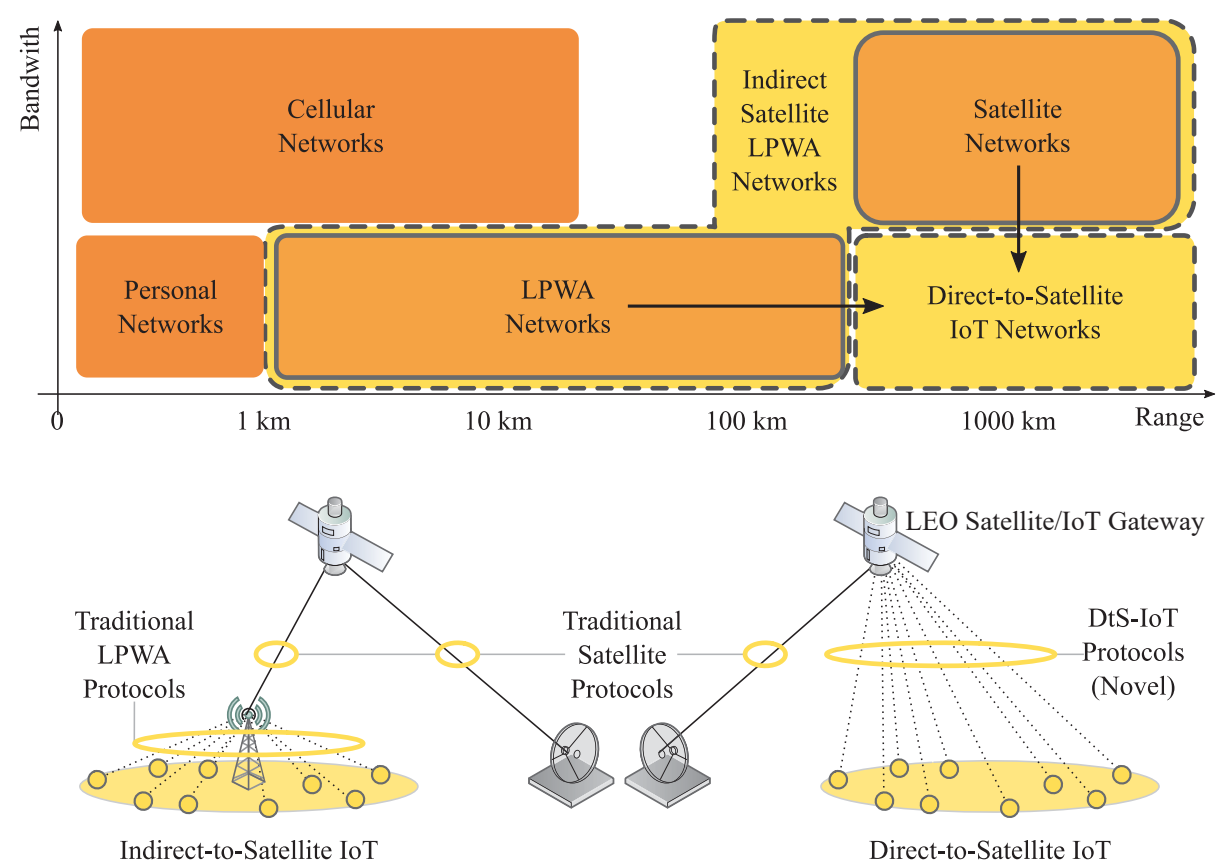

Fig. 1. Required bandwidth vs. range capacity for personal, cellular, LPWA and satellite networks. Direct Satellite LPWA domain is highlighted as an open research area requiring novel protocol design and/or adaptation of existing ones.

are two modes of interoperability envisioned for such a link: direct access and indirect access. The direct access mode allows devices to directly communicate with the satellite [29], while in the indirect access mode, each sensor and actuator in a network may communicate with the satellite through an intermediate sink node [11], i.e., the LPWA gateway in our scenario of study. Such a gateway is equipped with a traditional satellite terminal and a traditional LPWA radio interface to communicate with the sensor or actuator nodes in the area. On the one hand, the existing protocols can be leveraged in indirect mode, with the limitation that the area of a deployment is confined to the coverage of the gateway node on ground. On the other hand, a direct access from the sensor and actuator terminals to the satellite is a more appealing solution in challenging scenarios, for example: $(\boldsymbol{i})$ in disaster areas where rapid and infrastructure-less deployments are required, $(\boldsymbol{i i})$ in areas with very low device density where a gateway solution is not profitable, and (iii) in areas where devices will be present for a limited period of time and thus a gateway placement is discouraged.

However, existing LPWA protocols need to be revised as they were not designed to operate over several hundreds of kilometers in a ground-to-space link. Similarly, existing satellite protocols were thought to operate on highly directional point-to-point topologies and might not be fitted for LPWA applications. 
In this paper, we study the Direct-to-Satellite IoT (DtS-IoT) architecture as a novel networking domain in the intersection of traditional satellite networking and LPWA IoT networks, as illustrated in Figure 1. We present a thorough overview of existing protocols and their limitations to serve DtS-IoT, to then survey existing research on the physical, link, and upper layers of this new networking area. We will specifically focus on LoRa technologies and argue that, despite a few relevant considerations, it can be adapted to fit the requirements of DtS-IoT.

To help the reading, we briefly sketch how this contribution is structured. In Section 2 we revise existing protocols in the satellite and LPWA areas and discuss their limitation to efficiently operate in DtS-IoT network. In Section 3 we provide a detailed survey of specific research of potential application in a DtS-IoT architecture. Section 4 discusses the challenges and considerations of DtS-IoT satellite constellations. Section 5 concludes and summarizes this survey and points towards future research directions to realize future DtS-IoT systems.

\section{Existing Protocols}

\subsection{Satellite Network Protocols}

Many of existent satellite protocols for scientific and Earth Observation missions are standardized by the Consultative Committee for Space Data Systems (CCSDS). However, these protocol set are not thought for networking hundreds of devices on sight. Commercial applications on this domain typically rely on proprietary protocols, while only a few support Internet protocols. The most representative protocols are discussed hereafter, and their applicability challenges are highlighting with respect to the DtS-IoT domain.

Multiple channel access In the multiple channel access coordination, the standard DVB-RCS2 defines both contention-free and contention-based multiple access. Other commercial systems employ ALOHA-based protocols suitable for specific traffic patterns. A quantitative evaluation of traditional satellite Medium Access Control (MAC) protocols in DtS-IoT scenarios with LEO constellations is provided in [23].

DVB-RCS2: To support contention-based access, the standard provides two forms of random access methods: Slotted Aloha and Contention Resolution Diversity Slotted Aloha (CRDSA) [17]. The latter groups time-slots to form a frame and nodes send two or more copies of a packet, to later resolve the collisions using of a successive interference cancellation mechanism [13,18]. As for contention-free access, the standard defines the use of a Multi-Frequency Time Division Multiple Access (MF-TDMA) over the downlink channel, which in turn coordinates channel assignments by means of the Demand Assigned Multiple Access (DAMA) protocol [17]. Although high efficiency return channels can be adapted to the machine-to-machine communication services [18], they have been 
focused in hundreds of user terminals to provide remote Internet connectivity, but not for low power low datarate IoT terminals. Besides, time synchronization in LEO constellation-based IoT system is complex to achieve given the relative high motion between the satellites and ground devices [47].

Enhanced Aloha: This is a protocol operating in commercial telemetry satellite systems that exploits the periodicity of monitoring traffic sent by sensor nodes dedicated to Earth observation, scientific and environmental research. E-Aloha introduces a time window around the fixed sending times to reduce collisions among nodes attempting to transmit at the same time intervals. It is a simplified version of Aloha with no reliability features or additional control to avoid collisions [35]. Although part of the IoT traffic is well represented by periodic data transmission, there is also event-triggered transmissions with random generation patterns that will not be well-suited for the specific design of the E-Aloha protocol [29].

CubeSat Protocols: CubeSat deployments often employ frequencies in the range of the amateur frequency band, with very low data rates (i.e., in the order of $9.6 \mathrm{kbps}$ to $100 \mathrm{kbps}$ ). Although other higher bands are also explored in recent developments, the CubeSat deployments are characterized by low data rates and restricted contact times [17]. Traditional satellite protocols tend to behave poorly or simply do not work on such constrained devices. As a result, specific CubeSat protocols to serve the specific characteristics of the IoT traffic has been studied in the literature. In [23], it has been identified that advanced techniques such dynamic channelling or precise channel estimation (employed for interference cancellation), require costly resources often not available in low-cost CubeSat deployments. Among the random access MAC protocols evaluated, only a few become near the region where DtS-IoT supported by CubeSats provides a scalable, energy-efficient, and non-complex channel access mechanism to the ground sensor nodes.

Upper-layers satellite protocols The support of IP is a requirement if network segments connected via satellite networks interact with the Internet. This is naturally well-supported in satellite systems providing Internet Broadband access; however, that is not necessarily the case for more isolated satellite sensor networks. One would expect that IP support is a must in satellite IoT networks. Currently, the DVB-RCS2 standard supports IPv6 with a generic stream encapsulation (GSE) [17]. In the case of transport layer protocols, a customization of TCP stack parameters has been defined back in the late 90's to improve the performance over satellite links [6]. With TCP support, it is possible to employ traditional application layer protocols such as HTTP and also MQTT. More recent IoT protocols such as CoAP, which relies on UDP, have been evaluated over disruptive satellite links, showing the need to adjust the protocol parameters to achieve reduced end-to-end delays and to increase the packet delivery rate [26]. 


\subsection{LPWA Network Protocols}

NB-IoT: it is an IoT technology set up by the 3GPP as a part of Release 13 [32]. Although it is integrated into the Long-Term Evolution (LTE) standard, it can be regarded as a new air interface that operates on licensed radio spectrum [16]. Licensed band spectrum auctions of the sub-GHz spectrum are typically over 500 million dollars per $\mathrm{MHz}$ [33]. NB-IoT may operate as a dedicated carrier, it may occupy bandwidth of a wide-band LTE carrier, or it may use the guardbands of the LTE carrier [47]. Such a technology is also capable of guaranteeing higher datarates than common LPWA technologies, thus perfectly fitting disaster scenarios, where capturing videos/images would be needed for fine-grained control. If this capability perfectly match an emergency situation, it results as oversized (and expensive) for regular monitoring in normal situations. Eventually, although previous works has discussed the applicability of LTE over satellite links [42], they require complex coding and synchronous signaling protocol techniques which are not suitable for resource-constrained DtS-IoT devices [51].

LoRa: it is the LPWA technology getting the widest interest into the research community on IoT communications, for several reasons. LoRa works on unlicensed spectrum supporting an asynchronous bidirectional link layer protocol defined in the open LoRaWAN specification [3]. More interestingly, it implements roaming by allowing IoT devices to communicate to redundantly deliver information through all LoRa gateways in range. The LoRa physical layer uses the Chirp Spread Sprectrum (CSS) modulation to handle interference, and multipath fading, but it cannot offer the same Quality of Service that NB-IoT or LTE can provide over a licensed spectrum [51]. However, LoRa may have a wider network coverage than NB-IoT network. For example, in Belgium, a country with a total area of approximately $30500 \mathrm{~km} 2$, the LoRa network deployment covers the entire country with only seven base stations [33]. However, LoRa physical layer is a proprietary technology, which might impose economical constraints if considered for DtS-IoT. At the link layer, LoRa defines a lightweight protocol, namely LoRaWAN, permitting different modes of operation [34]. In the most typical scenario, when the application running on a low power device intends to deliver some data through the Internet, it turns the radio on for the exact time needed to perform the frame transmission. As matter of facts, this mode of communication, namely Class-A, is an asynchronous Pure ALOHA-based protocol, and provides the maximum battery life-time for sensors and must be supported by all LoRaWAN devices. Bidirectional traffic is supported by a receive window which is opened two times exactly $1 \mathrm{~s}$ and $2 \mathrm{~s}$ after the end of the uplink transmission. Notably, downlink unicast transmissions are synchronous with the reception of an uplink frame by the intended recipient device. To allow asynchronous downlink communications, Class-B devices must first listen to beacons broadcasted by gateways. In such a way, they get synchronized to the network and can safely open receiving windows at regular times. Downlink frames can be received during such time windows. Interestingly, the use of slotted Aloha or collision-free protocols can be enabled through proper protocol enhancement [3]. 
Finally, Class $\mathrm{C}$ devices are the main powered actuators that have sufficient power available to sustain a continuous receive window.

Given the few available applicability studies of LoRa technology over the satellite link, and the lack of performance evaluations of the LoRaWAN MAC protocols over DtS-IoT [41, 23], it remains largely unknown if these protocols are suitable for the scenario of study. Moreover, the IoT standards did not consider any satellite segment in the overall architecture, nor satellite standards considered low-power devices on the ground segment. This derives in the emergence of a new area of research as illustrated in Figure 1.

\section{Direct-to-Satellite IoT}

To successfully realize direct-to-satellite IoT, underlying protocol layers should be revised and adapted as necessary. In this context, we analyze Physical, Link and Upper Layers and they role in DtS-IoT. A cross-layer approach is not discarded as a means to achieve DtS-IoT.

Recent studies have shown the feasibility of using LPWA technologies over the satellite link for DtS-IoT architectures. In [47], Qu et al. explore the spectrum sharing of LEO satellite IoT constellation with terrestrial IoT systems such as LoRa and NB-IoT. Other experimental works defined LoRa performance over long distance links up to $250 \mathrm{~km}$, which could be considered the distance for a LEO satellite deployment [19]. In [21], the authors established the Doppler effects over links lengths under and above $550 \mathrm{~km}$, with a differentiated behavior that contradicts previous studies. The experiments were carried out in the laboratory and outdoors (with cars and line-of-sight conditions), using similar velocities and reproducing the Doppler effect over the link with software-defined-radios [21]. In all cases, the resulting data-rate at long distances is extremely low, enabling messages of a few bits, which indeed, are still valuable for many DtSIoT applications.

In the following, we discuss the upcoming challenges at each layer to enable DtS-IoT with LPWA technologies.

\subsection{Physical Layer}

Frequency Spectrum: LoRa has been designed to be used while complying within the unlicensed $900 \mathrm{MHz}$ Industrial, Scientific and Medical (ISM) frequency band in South America and within the unlicensed $868 \mathrm{Mhz}$ ISM frequency band in Europe, while NB-IoT was designed to be used in licensed $3 \mathrm{G} / 4 \mathrm{G}$ spectrum. Sharing the spectrum with terrestrial IoT systems will cause interference on both satellite and terrestrial segments, especially when large areas are covered by the satellite LEO system. Additional anti-interference measures are needed to enable the co-existence of these systems [47].

Multi-Beam Antennas: In order to deliver broadband data services, satellite systems have traditionally allowed to reuse the available bandwidth in beams with 
different frequencies, each serving one user. This scheme is known as frequency division multiplexing (FDM) [27]. Compared with a single global beam transmission, the use of a multi-beam architecture can bring several advantages, such as sending different symbols simultaneously to geographically separated areas. This indeed enabled an increment in the overall user bandwidth [28]; however, frequency reuse schemes cannot dramatically increase the channel capacity [54]. To further improve the spectral efficiency, full frequency reuse has been applied to modern multi-beam satellite systems, where interference mitigation techniques are implemented to mitigate interference between beams [56]. Multi-beams antennas will indeed become an appealing feature to provide enough channel diversity in DtS-IoT with thousands of devices at sight. However, technologies of hundreds of beams might be necessary to implement massive DtS-IoT.

Spread Spectrum Modulation: Signal transmissions for low-power devices have been studied on two fronts: spread-spectrum and narrow-band. Spread-spectrum techniques [44] consists in spreading the signal into a wider bandwidth, which render a good tolerance to interference. For example, LoRa leverages a unique chirp spread spectrum modulation (CSS), also defined as Direct-Sequence Spread Spectrum [10], to achieve exceptional link budget and low power performance within contested ISM channels. As shown in Figure 2 a), symbols are represented as instantaneous changes in the frequency of a chirp. However, recent studies have demonstrated that LoRa modulation may introduce difficulties in decoding signals from multiple terminals when used over satellite links. The authors in [45] propose another modulation called symmetric CSS that addresses this problem. Moreover, the LoRa physical layer is closed source and proprietary, thus there is no official references or protocol specifications, which might hinder its effective and immediate application to the satellite domain. The interested reader is referred to the work in [30], which provides a blind analysis of the protocol.

Narrow Band Modulation: Other systems use the classical transmission scheme with a signal being transmitted on a carrier of very small bandwidth (less than $1 \mathrm{kHz}$ ), a.k.a. ultra-narrow-band transmission. With this scheme it is possible to use simpler transceiver than in the case of spread spectrum techniques [7]. It provides natural resistance to noise and interference, as well as long range communications, and the terminal can be implemented with very low cost electronic components with low power consumption [31]. However, small frequency variations over time may become relatively large compared to the signal bandwidth (up to several times the frequency bandwidth of the signal). This frequency drift is particularly present in the case of a LEO satellite. As illustrated in Figure 2 b), for a satellite at an altitude of $720 \mathrm{~km}$, the Doppler rate can be as high as $-100 \mathrm{~Hz} / \mathrm{s}$ for a carrier frequency of $400 \mathrm{MHz}$. Authors in [7] state that, to tolerate such frequency drifts, narrow-band access scheme should be considered a random access. 

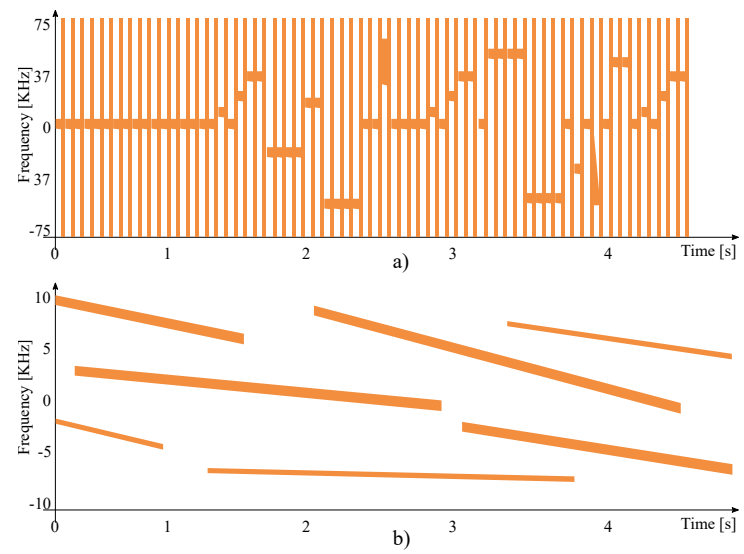

Fig. 2. Narrow-band and spread-spectrum illustration. (a) Chirp-based LoRa spreadspectrum signals occupying a channel of $125 \mathrm{KHz}$ [30]. (b) Narrow-band signals of 100 $\mathrm{Hz}$ wide and 2.5 seconds long, with different frequency shifts [7].

\subsection{Link Layer}

Link layer duties such as error correction and detection in a DtS-IoT system are rather not modified with respect to what was discussed for general space-toground communications in Section 2.1. However, the main novelty in DtS-IoT is the need to control the channel access among thousands of devices to satellites over hundreds of kilometers. The challenges in designing link layer MAC protocols in satellite IoT contexts are surveyed in [17] and [23]. In general, when energy efficiency is very important, TDMA is the well suited multiple channel access scheme. On the other hand, in many IoT deployments featured by a very high numbers of devices, and a limited portion of them having data to send, a fixed-assignment multiple access scheme such as TDMA might be inefficient [17]. Nevertheless, contention-based protocols, when used in the DtS-IoT context, need to integrate mechanisms to manage the links power imbalances, the uneven link delays, and the lack of high quality channel estimations [23]. In the remaining part of this subsection, we review recent proposals of MAC layer protocols suitable for DtS-IoT architectures.

Contention-Free Direct Access The algorithm proposed in [29] allows a satellite to collect data efficiently from sensor nodes through direct access. According to this scheme, the satellite allocates time slots on demand to sensor nodes which have data to send relying on a method consisting of a searching phase and an allocation phase. In the searching phase, the satellite finds the sensor nodes having data to send and divides the sensor nodes into two groups, and iterates until no more groups can be created. In the allocation phase, the satellite allocates time slots to all the sensor nodes of the remaining groups. Authors proved that this algorithm achieves higher efficiency in bandwidth utilization 
with respect to a TDMA-based fixed assignments scheme or a slotted ALOHA scheme discussed in Section 2.1.

Contended Direct Access: There are many random access techniques proposed in the literature $[2,49,14,12,43,18]$. Specifically, authors in [7] studied a version of Aloha extended for ultra-narrow-band signals, called Time/Frequency Aloha (TFA). In TFA, the frequency is chosen randomly within a specific bandwidth by the device. The analysis shows that the frequency drift in DtS-IoT narrowband increases the probability of collision, drastically limiting MAC performance beyond a network load of 0.2. Other MAC protocol for sensor data collection satellite systems using LoRa technology over the satellite link is proposed in [20]. The authors propose to condense both the LoRa gateway and the network server on the satellite. They introduce a delay function to reduce collisions among terminal nodes, at the same time that define a power adaptation technique based on the location of sensor nodes. The protocol was evaluated in a simulated environment with several simplifications that require further improvements to verify the performance on more realistic satellite scenarios.

\subsection{Upper Layers}

Addresses: IPv6 has increased the number of addresses that can be used throughout Internet. Addressing huge numbers of nodes on the ground with IPv6 is then feasible within DtS-IoT systems, as analyzed in [17]. At the standardization level, there is an open issue about how to provide IP addressing capabilities to more and more constrained devices in more and more dense deployments. One major limitation of LPWA technologies (as well as DtS-IoT) is the reduced MTU, which creates the need to implement middle boxes (i.e., a gateway) to interface non-IP technologies with the IP world. If the backhaul of DtS-IoT architectures is implemented with LPWA technologies over the satellite link, standards for packet compression and packet fragmentation become relevant to enable IP support up to the end device [37]. Given the hub-and-spoke nature of LPWA networks, other IPv6-related standards such as Neighbor Discovery help in achieving a stateful compression of the IPv6 header [52]. Another aspect to consider is that LEO constellation are in facts mobile networks, with the infrastructure moving with respect to the ground nodes (that may be static). In such a case, the context of communications at the IP level may change. A study of dynamic contexts combined with IP packet compression is explored in [1]. Further research and experimentation of the IP support (and possibly the IP mobility management) over LPWA links in a satellite environment is required to understand the impact over the performance of both time-critical and delay tolerant IoT applications.

Broadcast and Anycast: Several applications for IoT will exploit DtS-IoT forward link for delivering commands and control information via satellite to the huge number of devices [4]. In emergency management systems, for example, the forward link communication could be used for providing group oriented services to support both remote sensors or actuators and human-type communications [24]. 
To efficiently exploit the large coverage provided by satellite systems, efficient broadcast and localized anycast will need to be supported. Moreover, geocast, or geographically localized distribution of messages are key features to motivate the deployment of future DtS-IoT systems.

Transport and Application layer protocols: Two of the most promising application layer protocols for small devices are MQTT and CoAP. Both are open standards better suited to constrained environments than HTTP, provide mechanisms for asynchronous communication, and run on IP, which facilitates their integration within Internet. On the one hand, MQTT was proposed by IBM implements a publish/subscribe paradigm and is TCP-based. MQTT gives flexibility in communication patterns and acts purely as a pipe for binary data. On the other hand, IETF has proposed the use of Constrained Application Protocol (CoAP) (RFC 7252), which relies on UDP and implements a request/response style. When used over satellite links, CoAP outperforms MQTT on random access channels $[9,8]$. Moreover, the authors of [39] proposed an integration of CoAP and MQTT over LoRaWAN, even though such application layer protocols cause extra overhead in terms of payload size, thus undermining the already limited performance of DtS-IoT links.

\section{DtS-IoT Constellations}

Depending on the satellite orbit and device latitude, a typical LEO satellite provides an average of 4 passes per day. Each pass, depending on the satellite altitude, offers data transfer opportunities in between 7 and 10 minutes duration when the satellite flies exactly over the spot on ground. When the satellite passes closer to the horizon from the device perspective, the contact duration is reduced and the channel conditions are worsened. This condition renders connectivity times in the order of 20 minutes per location per day, on which the channel resource will likely be shared among hundreds if not thousands of devices. Because of the limited data rate of a DtS-IoT link, the data transfer provided by a single satellite might result insufficient for a typical IoT application.

Satellites constellations are fleets of LEO satellites strategically distributed in orbit to provide continuous or quasi-continuous coverage of the whole planet's surface, or part of it. As a result, constellations enhance the overall data transfer and reduces the waiting time to access the network. For example, the constellation in Figure 3 a) provides world coverage by leveraging polar orbits (i.e., orbits with 90 degree inclination that passes over the poles). Figure $3 \mathrm{~b}$ ), on the other hand, provides better revisit of satellites over populated areas near the equator; but inclined orbits never flies on the poles rendering a partial planet coverage. There is not one-fits-all constellation topology configuration, a trade-off is always present between surface coverage and satellite revisit time.

In general, the application drives the constellation parameters. For example, voice and Internet data services constellation (i.e., Iridium) are configured to always have at least one satellite covering every telephone or Internet device on 


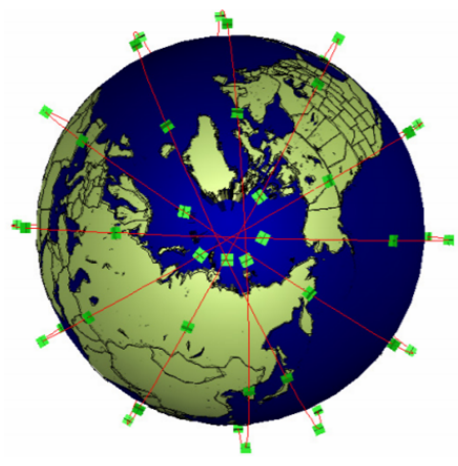

a)

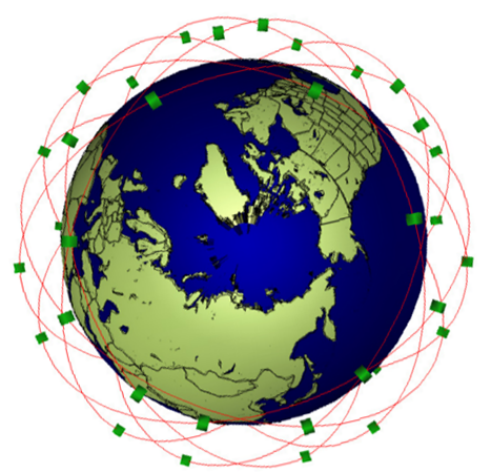

b)

Fig. 3. LEO satellite constellation with a Walker Star formation on a) and a Walker Delta (a.k.a. Ballard Rosette) on b). Iridium constellation uses the former to provide world-wide connectivity including the poles, while Globalstar is based on the latter, which provides better coverage in populated areas. Images created with $\mathrm{SaVi}$ [53]

the Earth surface. A DtS-IoT application could indeed profit from a full-coverage constellation topology as devices could be permanently at sight of a passing satellite. However, continuous coverage is achieved at the expense of hundreds of LEO satellites, if not more. This can certainly make prohibitive the cost of the fleet for many IoT applications, which are designed to be predominantly low-cost.

Another possibility is to consider constellations with less satellites that provides partial and opportunistic connectivity. This is indeed an intermediate solution between a single LEO satellite and a full-coverage constellation. In this case, devices would need to store data until one of the constellation's satellite becomes reachable. These sparse constellations would provide a higher latency service, but at a reduced deployment and operation cost. This approach is likely to satisfy a large set of IoT applications requirements.

From an IoT protocol perspective, full-coverage constellations can provide continuous connectivity mimicking the permanent presence of an IoT gateway for all the serviced devices on ground. In the case of LoRa, a continuous gateway presence would facilitate a class A operation mode, the default mode for LoRa devices. However, a sparse constellation would require devices to sporadically operate in the so-called "beacon-less" mode in class B [34], when no satellite is on sight. In particular, devices are allowed to sustain a class B operation for two hours (120 minutes) after receiving the last beacon, in this case, from a DtS-IoT satellite. During this period, the device would need to rely on its local clock to keep timing.

As a result, a sparse DtS-IoT constellation design would need to consider such timing constraints not present in other types of satellite constellations. Furthermore, a discussion remains open on the feasibility of enabling DtS-IoT devices 
with simplified orbital information to only enable the radio when a satellite is on sight, a fundamental DtS-IoT battery saving feature. The effective DtS-IoT constellation topology design as well the implications on the specific protocol adaptations is left as an immediate future work.

\section{$5 \quad$ Summary and Outlook}

As we move towards an all-connected era, IoT networking technologies will need to embrace satellite as a means to connect remote devices over long-range, lowpower and low data-rate. While indirect approaches can leverage existing satellite and LPWA protocols, a direct connection to devices on ground offers a real remote IoT experience.

In this paper, we have introduced and described Direct-to-Satellite-IoT as a promising research area where existing protocols need to be revised and adapted. A survey on relevant technologies showed that LoRa is an appealing approach towards DtS-IoT, but imposes severe challenges motivating future research efforts. Among them, we have highlighted the extremely low data-rate, the lack of knowledge on how the satellite channel affects the behavior of a proprietary technology, and the need of IP support for integration with Internet. We have also identified DtS-IoT constellations as a promising solution with pending challenges to tackle at a protocol level.

Future research is envisioned in developing a new LoRa-compatible interface including new MAC protocols designed for the low-power consumption but considering the satellite system, especially if deployed in constellations. Moreover, architectural decisions involving the satellites will need to be discussed. In particular, the LoRaWAN gateway and server roles might need to be distributed among satellites in order to get rid of the dependency of the network server for both data and control planes. To this end, the fact that gateways will be moving at high speeds will need to be studied and the role of routing via inter-satellite links will need to be analyzed in detail. Finally, upper layer transport and application protocols (CoAP and MQTT) will be evaluated over the DtS-IoT links.

\section{References}

1. Abdelfadeel, K.Q., Cionca, V., Pesch, D.: Dynamic context for static context header compression in LPWANs. In: Proceedings - 14th Annual International Conference on Distributed Computing in Sensor Systems, DCOSS 2018 (2018). https://doi.org/10.1109/DCOSS.2018.00013

2. Abramson, N.: The aloha system: another alternative for computer communications. Fall Joint Computer Conference 37, 281-285 (01 1977). https://doi.org/10.1145/1478462.1478502

3. Accettura, N., Alata, E., Berthou, P., Dragomirescu, D., Monteil, T.: Addressing scalable, optimal, and secure communications over LoRa networks: Challenges and research directions. Internet Technology Letters 1(4), e54 (jul 2018). https://doi.org/10.1002/itl2.54, http://doi.wiley.com/10.1002/itl2.54 
4. Afolabi, R.O., Dadlani, A., Kim, K.: Multicast scheduling and resource allocation algorithms for ofdma-based systems: A survey. IEEE Communications Surveys Tutorials 15(1), 240-254 (First 2013). https://doi.org/10.1109/SURV.2012.013012.00074

5. Agarwal, A., Gupta, S., Kumar, S., Singh, D.: An efficient use of iot for satellite data in land cover monitoring to estimate lst and et. In: 2016 11th International Conference on Industrial and Information Systems (ICIIS). pp. 905-909 (Dec 2016). https://doi.org/10.1109/ICIINFS.2016.8263067

6. Allman, M., Glover, D., Sanchez, L.: Enhancing tcp over satellite channels using standard mechanisms. BCP 28, RFC Editor (January 1999)

7. Anteur, M., Deslandes, V., Thomas, N., Beylot, A.: Ultra narrow band technique for low power wide area communications. In: 2015 IEEE Global Communications Conference (GLOBECOM). pp. 1-6 (Dec 2015). https://doi.org/10.1109/GLOCOM.2015.7417420

8. Bacco, M., Boero, L., Cassara, P., Colucci, M., Gotta, A., Marchese, M., Patrone, F.: Iot applications and services in space information networks. IEEE Wireless Communications 26(2), 31-37 (April 2019). https://doi.org/10.1109/MWC.2019.1800297

9. Bacco, M., Colucci, M., Gotta, A.: Application protocols enabling internet of remote things via random access satellite channels. In: 2017 IEEE International Conference on Communications (ICC). pp. 1-6 (May 2017). https://doi.org/10.1109/ICC.2017.7997292

10. Berni, A., Gregg, W.: On the utility of chirp modulation for digital signaling. IEEE Transactions on Communications 21(6), 748-751 (June 1973). https://doi.org/10.1109/TCOM.1973.1091721

11. Bisio, I., Marchese, M.: Efficient Satellite-Based Sensor Networks for Information Retrieval. IEEE Systems Journal 2, 464-475 (Dec 2008). https://doi.org/10.1109/JSYST.2008.2004850

12. Casini, E., De Gaudenzi, R., Del Rio Herrero, O.: Contention resolution diversity slotted aloha (crdsa): An enhanced random access schemefor satellite access packet networks. IEEE Transactions on Wireless Communications 6(4), 1408-1419 (April 2007). https://doi.org/10.1109/TWC.2007.348337

13. Casini, E., De Gaudenzi, R., Del Rio Herrero, O.: Contention resolution diversity slotted ALOHA (CRDSA): An enhanced random access scheme for satellite access packet networks. IEEE Transactions on Wireless Communications 6(4), 1408-1419 (2007). https://doi.org/10.1109/TWC.2007.348337

14. Choudhury, G., Rappaport, S.: Diversity aloha - a random access scheme for satellite communications. IEEE Transactions on Communications 31(3), 450-457 (March 1983). https://doi.org/10.1109/TCOM.1983.1095828

15. Cocco, G., Ibars, C.: On the feasibility of satellite $\mathrm{m} 2 \mathrm{~m}$ systems. In: 30th AIAA International Communications Satellite System Conference (ICSSC) (09 2012). https://doi.org/10.2514/6.2012-15074, https://arc.aiaa.org/doi/abs/10.2514/6.2012-15074

16. D. Rohde, J. Schwarz: Narrowband Internet of Things, Aug., 2016. (2016 August), available: https://www.rohde-schwarz.com/us/applications/narrowband-internetof-things-application-note 56280-314242.html

17. De Sanctis, M., Cianca, E., Araniti, G., Bisio, I., Prasad, R.: Satellite communications supporting internet of remote things. IEEE Internet of Things Journal 3(1), 113-123 (Feb 2016). https://doi.org/10.1109/JIOT.2015.2487046 
18. Del Rio Herrero, O., De Gaudenzi, R.: High efficiency satellite multiple access scheme for machine-to-machine communications. IEEE Transactions on Aerospace and Electronic Systems 48(4), 2961-2989 (October 2012). https://doi.org/10.1109/TAES.2012.6324672

19. Demetri, S., Zúñiga, M., Picco, G.P., Kuipers, F., Bruzzone, L., Telkamp, T.: Automated Estimation of Link Quality for LoRa : A Remote Sensing Approach. In: Proceedings of the 18th International Conference on Information Processing in Sensor Networks. pp. 145-156. ACM, Montreal (2019), https://dl.acm.org/citation.cfm?doid=3302506.3310396

20. Deng, T., Zhu, J., Nie, Z.: An adaptive mac protocol for sdcs system based on lora technology. In: 2017 2nd International Conference on Automation, Mechanical Control and Computational Engineering (AMCCE 2017). Atlantis Press (2017/03). https://doi.org/https://doi.org/10.2991/amcce17.2017.146, https://doi.org/10.2991/amcce-17.2017.146

21. Doroshkin, A., Zadorozhny, A., Kus, O., Prokopyev, V.: Experimental Study of LoRa Modulation Immunity to Doppler Effect in CubeSat Radio Communications. IEEE Access PP(c), 1 (2019). https://doi.org/10.1109/ACCESS.2019.2919274

22. Fairhurst, G., Caviglione, L., Collini-Nocker, B.: First: Future internet - a role for satellite technology. In: 2008 IEEE International Workshop on Satellite and Space Communications. pp. 160-164 (Oct 2008). https://doi.org/10.1109/IWSSC.2008.4656774

23. Ferrer, T., Céspedes, S., Becerra, A.: Review and Evaluation of MAC Protocols for Satellite IoT Systems Using Nanosatellites. Sensors 19(8), 1947 (apr 2019). https://doi.org/10.3390/s19081947, https://www.mdpi.com/1424$8220 / 19 / 8 / 1947$

24. Franck, L., Suffritti, R.: Multiple alert message encapsulation over satellite. In: 2009 1st International Conference on Wireless Communication, Vehicular Technology, Information Theory and Aerospace Electronic Systems Technology. pp. 540-543 (May 2009). https://doi.org/10.1109/WIRELESSVITAE.2009.5172503

25. Ghavimi, F., Chen, H.: M2m communications in 3gpp lte/lte-a networks: Architectures, service requirements, challenges, and applications. IEEE Communications Surveys Tutorials 17(2), 525-549 (Secondquarter 2015). https://doi.org/10.1109/COMST.2014.2361626

26. Giotti, D., Lamorte, L., Soua, R., Palattella, M.R., Engel, T.: Performance Analysis of CoAP under Satellite Link Disruption. In: 2018 25th International Conference on Telecommunications (ICT). pp. 623-628. IEEE, St. Malo (2018). https://doi.org/10.1109/ICT.2018.8464881

27. $\mathrm{Hu}, \mathrm{D} ., \mathrm{He}, \mathrm{L} ., \mathrm{Wu}, \mathrm{J} .:$ A novel forward-link multiplexed scheme in satellite-based internet of things. IEEE Internet of Things Journal 5(2), 1265-1274 (April 2018). https://doi.org/10.1109/JIOT.2018.2799550

28. Joroughi, V., Vázquez, M.Á., Pérez-Neira, A.I.: Generalized multicast multibeam precoding for satellite communications. IEEE Transactions on Wireless Communications 16(2), 952-966 (Feb 2017). https://doi.org/10.1109/TWC.2016.2635139

29. Kawamoto, Y., Nishiyama, H., Fadlullah, Z.M., Kato, N.: Effective data collection via satellite-routed sensor system (srss) to realize global-scaled internet of things. IEEE Sensors Journal 13(10), 3645-3654 (Oct 2013). https://doi.org/10.1109/JSEN.2013.2262676

30. Knight, M., Seeber, B.: Decoding lora: Realizing a modern lpwan with sdr. Proceedings of the GNU Radio Conference 1(1) (2016), https://pubs.gnuradio.org/index.php/grcon/article/view/8 
31. Lassen, T.: Long-range rf communication: Why narrowband is the de facto standard. In: Texas Instruments White Paper (2014)

32. Link Labs, Inc.: A Comprehensive Look at Low Power, Wide Area Networks, available: http://cdn2.hubspot.net/hubfs/427771/LPWAN-Brochure-Interactive.pdf.

33. LoRa Alliance: LoRaWAN What is it. Technical Marketing Workgroup 1.0, Nov., 2015. (November 2015), available: https://www.loraalliance.org/portals/0/documents/whitepapers/LoRaWAN101.pdf.

34. LoRa Alliance Technical Committee: LoRaWAN ${ }^{\mathrm{TM}} 1.1$ Specification (Oct 2017), v1.1

35. Ma, H., Cai, L.: Performance analysis of randomized mac for satellite telemetry systems. In: 2010 5th International ICST Conference on Communications and Networking in China. pp. 1-5 (Aug 2010)

36. Meloni, A., Atzori, L.: The role of satellite communications in the smart grid. Wireless Commun. 24(2), 5056 (Apr 2017). https://doi.org/10.1109/MWC.2017.1600251, https://doi.org/10.1109/MWC.2017.1600251

37. Minaburo, A., Toutain, L., Gomez, C., Barthel, D., Zuniga, J.: Lpwan static context header compression (schc) and fragmentation for ipv6 and udp. Internet-Draft draft-ietf-lpwan-ipv6-static-context-hc-18, IETF Secretariat (December 2018), http://www.ietf.org/internet-drafts/draft-ietf-lpwan-ipv6-static-context-hc18.txt, http://www.ietf.org/internet-drafts/draft-ietf-lpwan-ipv6-static-contexthc-18.txt

38. Minoli, D.: Building the Internet of Things with IPv6 and MIPv6: The Evolving World of M2M Communications. Wiley Publishing, 1st edn. (2013)

39. Nguyen, T., Patonico, S., Bezunartea, M., Thielemans, S., Braeken, A., Steenhaut, K.: Horizontal integration of coap and mqtt on internet protocol based loramotes. In: 2018 IEEE 29th Annual International Symposium on Personal, Indoor and Mobile Radio Communications (PIMRC). pp. 1-7 (Sep 2018). https://doi.org/10.1109/PIMRC.2018.8580674

40. Palattella, M.R., Accettura, N., Vilajosana, X., Watteyne, T., Grieco, L.A., Boggia, G., Dohler, M.: Standardized protocol stack for the internet of (important) things. IEEE Communications Surveys Tutorials 15(3), 1389-1406 (Third 2013). https://doi.org/10.1109/SURV.2012.111412.00158

41. Palattella, M.R., Accettura, N.: Enabling Internet of Everything Everywhere: LPWAN with Satellite Backhaul. In: 2018 Global Information Infrastructure and Networking Symposium (GIIS). pp. 15. IEEE (oct 2018). https://doi.org/10.1109/GIIS.2018.8635663, https://ieeexplore.ieee.org/document/8635663/

42. Papaleo, M., Neri, M., Vanelli-Coralli, A., Corazza, G.E.: Using lte in 4g satellite communications: Increasing time diversity through forced retransmission. In: 2008 10th International Workshop on Signal Processing for Space Communications. pp. 1-4 (Oct 2008). https://doi.org/10.1109/SPSC.2008.4686699

43. Pateros, C.: Novel direct sequence spread spectrum multiple access technique. In: MILCOM 2000 Proceedings. 21st Century Military Communications. Architectures and Technologies for Information Superiority (Cat. No.00CH37155). vol. 1, pp. 564-568 vol.2 (Oct 2000). https://doi.org/10.1109/MILCOM.2000.905024

44. Pursley, M.B.: Direct-sequence spread-spectrum communications for multipath channels. IEEE Transactions on Microwave Theory and Techniques 50(3), 653661 (March 2002). https://doi.org/10.1109/22.989950 
45. Qian, Y., Ma, L., Liang, X.: Symmetry Chirp Spread Spectrum Modulation Used in LEO Satellite Internet of Things. IEEE Communications Letters 22(11), 2230-2233 (nov 2018). https://doi.org/10.1109/LCOMM.2018.2866820, https://ieeexplore.ieee.org/document/8444661/

46. Qu, Z., Zhang, G., Cao, H., Xie, J.: Leo satellite constellation for internet of things. IEEE Access 5, 18391-18401 (2017). https://doi.org/10.1109/ACCESS.2017.2735988

47. Qu, Z., Zhang, G., Cao, H., Xie, J.: LEO Satellite Constellation for Internet of Things. IEEE Access 5, 18391$18401 \quad$ (2017). https://doi.org/10.1109/ACCESS.2017.2735988, http://ieeexplore.ieee.org/document/8002583/

48. Rebbeck, T., Mackenzie, M., Afonso, N.: Low-powered wireless solutions have the potential to increase the $\mathrm{m} 2 \mathrm{~m}$ market by over 3 billion connections. In: Analysys Mason, London, UK (Sept 2014)

49. Roberts, L.: Aloha packet system with and without slots and capture. ACM SIGCOMM Computer Communication Review 5, 28-42 (04 1975). https://doi.org/10.1145/1024916.1024920

50. Sigfox: [Online] https://www.sigfox.com

51. Sinha, R.S., Wei, Y., Hwang, S.H.: A survey on lpwa technology: Lora and nb-iot. ICT Express 3(1), $14-21$ (2017). $\quad$ https://doi.org/https://doi.org/10.1016/j.icte.2017.03.004, http://www.sciencedirect.com/science/article/pii/S2405959517300061

52. Thubert, P.: Ipv6 neighbor discovery on wireless networks. Internet-Draft draft-thubert-6man-ipv6-over-wireless-01, IETF Secretariat (April 2019), http://www.ietf.org/internet-drafts/draft-thubert-6man-ipv6-over-wireless-01.txt, http://www.ietf.org/internet-drafts/draft-thubert-6man-ipv6-over-wireless-01.txt

53. Wood, L.: Savi: satellite constellation visualization (06 2011), available: https://savi.sourceforge.io/

54. Yu, Q., Meng, W., Yang, M., Zheng, L., Zhang, Z.: Virtual multibeamforming for distributed satellite clusters in space information networks. IEEE Wireless Communications 23(1), 95-101 (February 2016). https://doi.org/10.1109/MWC.2016.7422411

55. Zhang, N., Wang, M., Wang, N.: Precision agriculture-a worldwide overview. Computers and Electronics in Agriculture 36(2), 113 - 132 (2002). https://doi.org/https://doi.org/10.1016/S0168-1699(02)00096-0, http://www.sciencedirect.com/science/article/pii/S0168169902000960

56. Zheng, G., Chatzinotas, S., Ottersten, B.: Generic optimization of linear precoding in multibeam satellite systems. IEEE Transactions on Wireless Communications 11(6), 2308-2320 (June 2012). https://doi.org/10.1109/TWC.2012.040412.111629

57. Zhou, H.: The Internet of Things in the Cloud: A Middleware Perspective. CRC Press, Inc., Boca Raton, FL, USA, 1st edn. (2012) 